\title{
La formation des enseignants en Chine
}

Origine lointaine, évolution tortueuse et réformes récentes

Teacher training in China. Its distant origin, circuitous evolution and recent reforms

La formation de los docentes en China. Origen remoto, évolugción tortuosa y

reformas recientes

\section{Kechao Xing et Yajun Meng}

\section{OpenEdition \\ Journals}

Édition électronique

URL : http://journals.openedition.org/ries/2803

DOI : 10.4000/ries.2803

ISSN : 2261-4265

Éditeur

Centre international d'études pédagogiques

Édition imprimée

Date de publication : 1 mars 1999

Pagination : 85-92

ISBN : 9771254459005

ISSN : $1254-4590$

\section{Référence électronique}

Kechao Xing et Yajun Meng, "La formation des enseignants en Chine », Revue internationale d'éducation de Sèvres [En ligne], 21 | mars 1999, mis en ligne le 28 mai 2013, consulté le 06 janvier 2020. URL : http://journals.openedition.org/ries/2803 ; DOI : 10.4000/ries.2803

Ce document a été généré automatiquement le 6 janvier 2020

(c) Tous droits réservés 


\title{
La formation des enseignants en Chine
}

\author{
Origine lointaine, évolution tortueuse et réformes récentes \\ Teacher training in China. Its distant origin, circuitous evolution and recent \\ reforms \\ La formation de los docentes en China. Origen remoto, évolugción tortuosa y \\ reformas recientes
}

Kechao Xing et Yajun Meng

\section{Histoire et perspectives}

1 L'histoire de l'éducation en Chine est aussi longue que celle de sa civilisation. Déjà avec Xueji, un écrit paru plusieurs siècles avant notre ère traite d'une manière systématique tous les grands problèmes de l'éducation, dont la formation des enseignants. Cependant, durant les très longues années d'une société, féodale disent les uns, bureaucratique disent les autres, l'éducation restait le privilège des familles aisées et/ ou des personnes « douées » et était considérée comme l'un des moyens les plus sûrs de l'ascension sociale.

2 Dans cette situation, les magistrats paraissaient les mieux appropriés à enseigner et, à côté d'eux, c'étaient, pour la plupart du temps, des refusés aux concours de recrutement des mandarins qui enseignaient pour vivre. C'est la raison pour laquelle la formation des enseignants stricto sensu est restée longtemps marginale, sinon quasi inexistante en Chine et n'a jamais été mise à l'ordre du jour par aucun gouvernement. Les grands changements datent d'une période beaucoup plus récente où les pays occidentaux avaient déjà installé un système d'enseignement assez complet. Face à l'état arriéré du pays et à l'échec de "la formation des élites", des politiciens et éducateurs clairvoyants ont commencé, dès la fin du siècle dernier, des expériences historiques. Le premier département «normal » et la première «école normale » ont vu le jour respectivement à Shanghai en 1897 et à Jiangsu en 1902 et, presque en même 
temps, la formation des enseignants a fait officiellement et solennellement son apparition dans les deux premiers règlements impériaux établissant le système éducatif chinois de l'époque moderne (en 1901 et 1904, le second modifiant le premier qui n'a pas été mis en application). Combinant les expériences étrangères et la réalité chinoise, les documents fondateurs insistaient sur les principes suivants aujourd'hui encore valables pour l'essentiel :

- former un système fermé sur lui-même, échelonné sur deux niveaux et spécialisé dans des secteurs donnés (industriel, agricole et commercial);

- établir un examen d'entrée sévère, prendre en considération la formation morale, professionnelle et disciplinaire;

- établir la gratuité de la scolarité et l'obligation de servir l'État pendant un certain temps après les études ;

- recruter des formateurs qualifiés.

3 Vers la fin de la Dynastie des Qings (1908), on comptait en Chine une trentaine d'écoles normales avec plus de 5000 normaliens.

4 La Révolution de 1911 a mis fin à l'histoire des dynasties chinoises, pourtant le système de formation des enseignants, grâce à son caractère fondé, a été maintenu à quelques modifications près :

- une élévation de position par laquelle les écoles normales relevant autrefois des districts sont devenues provinciales ;

- un encouragement à la création des écoles normales pour les filles ;

- une planification unifiée à travers le pays des écoles normales supérieures qui avaient désormais leur étiquette nationale ;

- un développement de la recherche sur la formation des enseignants qui avait pour conclusion principale l'importance primordiale de cette formation et la nécessité d'élever le traitement des enseignants. Malgré la situation tumultueuse, sous la domination des seigneurs de la guerre, la formation a eu un développement lent mais stable pendant la première décennie de la République et entre 1912 et 1922, le nombre des écoles normales est passé de 253 à 385 et les élèves de 28525 à 43 846. Des écoles renommées ainsi que d'excellents normaliens se sont fait remarquer.

5 Pendant plus d'un demi-siècle, la formation des enseignants en Chine a connu des évolutions liées à l'histoire politique du pays :

- reflux des écoles normales causé par leur fusion avec les lycées et la relance du débat sur la nécessité de leur existence entre 1922 et 1928 ;

- nouvel essor après la publication d'une loi d'orientation de l'Éducation nationale entre 1929 et 1937 ;

- mesures provisoires de réparation pendant la guerre contre le Japon ;

- restitution et réorganisation, suivant le modèle de l'ex-Union soviétique, par la République populaire de Chine entre 1949 et 1956 ;

- recherche d'une voie propre à la Chine, sous l'influence de « gauche » entre 1956 et 1966 ;

- effondrement presque total de l'Éducation nationale au cours de la Révolution culturelle entre 1966 et 1976.

6 Le développement de la formation des enseignants en Chine n'a pu retrouver son cours normal qu'après la chute de la "Bande des Quatre », conformément à la réorientation de l'objectif général de tout le pays vers l'édification et le développement global de l'éducation nationale. 
Depuis, les réformes ont eu pour objectifs tout d'abord :

- de renforcer la législation et la réglementation comme garantie. L'Assemblée populaire nationale et le gouvernement ont promulgué une série de lois et de décrets concernant uniquement ou partiellement la formation des enseignants, dont :

- orientations du ministère de l'Éducation pour le développement de la formation des enseignants (1978),

- projets de programmes donnant suite à la réunion de travail sur la formation des enseignants (1980),

- orientations du ministère de l'Éducation pour la restructuration et la gestion du corps enseignant (1981),

décision du Comité central du Parti sur la réforme du système éducatif (1985),

- loi sur l'enseignement obligatoire (1986),

- règlement décrétant la carrière des enseignants et leur évaluation (1986),

- loi sur les enseignants (1993),

- règlement du Conseil d'État sur la qualification des enseignants (1995).

- ensuite, d'augmenter l'investissement comme support. Entre 1985 et 1995, le gouvernement central a alloué à la formation des enseignants, à part les dépenses ordinaires, une subvention spéciale de 2,1 milliards de yuans (230 millions d'euros) tout en y introduisant une somme de 245 millions d'euros de la part des organisations internationales (Unicef, Pnud, etc.), et ce avec une contrepartie de la part du bénéficiaire ;

- enfin, d'élever le niveau des actuels et futurs enseignants. On a essayé, d'une part, d'élever la qualification des enseignants du primaire et du secondaire par l'allongement de la scolarité pour les uns et le recyclage pour les autres et, d'autre part, d'améliorer la qualité de la formation en encourageant les meilleurs élèves à choisir ce métier et en introduisant dans l'enseignement de nouvelles matières et la recherche.

De 1980 à 1995, les écoles normales chinoises, secondaires et supérieures comprises, ont eu 5510000 diplômés, 4800000 enseignants en fonction ont suivi des cours aboutissant à un grade supérieur, le taux des enseignants au niveau primaire, collège et lycée ayant la qualification définie par l'État, est passé respectivement de 49,8 \% à 88,9\%, de 12,7\%, à 69,1\%, de 35,9\% à 55,2\%. Au cours du VIII Plan quinquennal (1991-1995), 7300000 directeurs d'écoles primaires et secondaires ont reçu une «formation à l'emploi » et ont désormais la qualification formellement requise pour assumer correctement leur fonction.

\section{Structures de formation}

9 Les établissements chinois constituant le réseau de formation des enseignants peuvent se distinguer par niveau et par secteur. Les écoles normales secondaires, une au moins par district, recrutent les diplômés des collèges, dispensent une formation de trois à quatre ans et ont pour tâche de former les enseignants du primaire. Une partie d'entre elles, intitulées écoles normales pour la petite enfance se spécialisent dans la formation des enseignants des jardins d'enfants qui sont, pour l'écrasante majorité, de sexe féminin. Les écoles normales supérieures de cycle court, au moins une par préfecture (autorité administrative chinoise entre district et province) recrutent les diplômés des lycées, dispensent une formation de deux à trois ans et ont pour tâche de former les enseignants des collèges, écoles secondaires du premier cycle. Les écoles normales supérieures de cycle normal (quatre années), appelées en Chine institut ou université 
normale au moins une par province, quelques-unes étant nationales dont la nôtre, forment les enseignants des lycées. Au même niveau, on trouve, à côté d'un grand nombre d'établissements à fonction commune, quelques institutions spécialisées, comme dans le secteur artistique, technique et professionnel, ainsi que pour les enfants handicapés. Les institutions de recyclage, écoles de recyclage et instituts d'éducation, se voient chargées de la formation continue des enseignants en poste. Parallèlement à ce réseau principal, existent d'autres formules complémentaires tels que les cycles de formation pour les futurs enseignants et les départements d'éducation dans d'autres établissements d'enseignement supérieur, les cours par correspondance ou par télévision, l'école du soir, l'examen après l'autodidaxie (voir tableau 1 ci-après).

\section{Structure de la formation}

Tableau 1

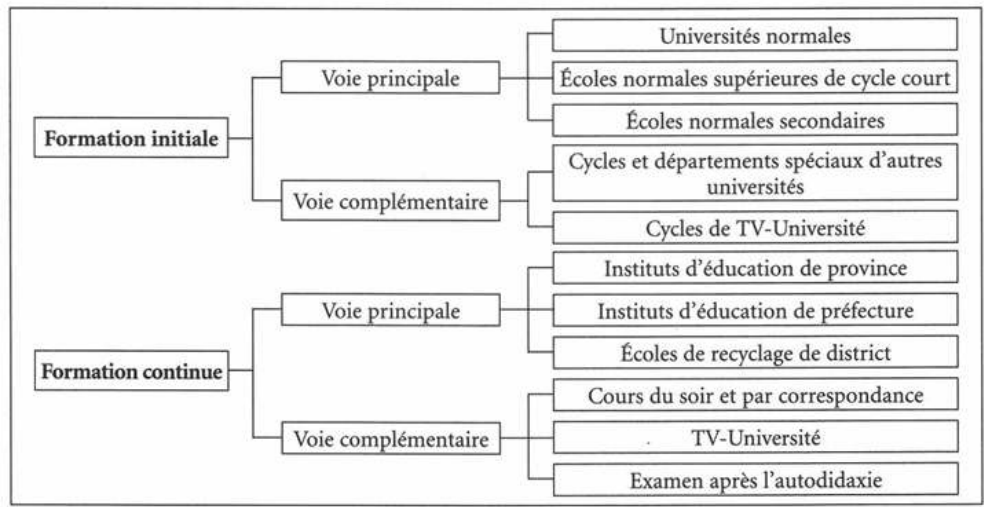

Données statistiques 1995

Tableau II

\begin{tabular}{|c|c|c|c|c|c|c|}
\hline & Établisssements & Elèves & Recrutés & Diplômés & Enseignants & $\begin{array}{c}\text { Non- } \\
\text { enseignants }\end{array}$ \\
\hline $\begin{array}{c}\text { Universités } \\
\text { normales } \\
\text { supérieures }\end{array}$ & 236 & 582000 & 210900 & 203900 & 72823 & 74021 \\
\hline $\begin{array}{c}\text { Ecoles } \\
\text { normales } \\
\text { sécondaires }\end{array}$ & 897 & 848000 & 308300 & 244800 & 61713 & 45677 \\
\hline $\begin{array}{c}\text { Instituts } \\
\text { d'éducation }\end{array}$ & 242 & 213700 & 77700 & 88300 & & \\
\hline $\begin{array}{c}\text { Écoles de } \\
\text { recyclage }\end{array}$ & 2031 & 516300 & 241500 & 214700 & & \\
\hline
\end{tabular}

\section{Contenus de la formation}

Si la législation, le financement et la structure constituent les conditions extérieures de la réussite pour la formation, néanmoins, les matières enseignées, leurs contenus, ainsi que la méthode restent primordiales. La formation initiale ${ }^{1}$ comprend trois groupes de matières enseignées dans les écoles normales chinoises, secondaires et supérieures: tronc commun, cours de la ou des disciplines de spécialité et formation pédagogique, dont la proportion varie selon le niveau et le secteur géographique.

11 À l'école normale secondaire où l'on forme les futurs enseignants du primaire, les élèves reçoivent les cours suivants :

- politique (une formation politique, idéologique et civique);

- langue et littérature chinoises ;

- mathématiques, physique, chimie, biologie, physiologie et hygiène ;

- histoire, géographie ;

- langue étrangère (anglais presque toujours) ; 
- psychologie, pédagogie, didactique du chinois, des mathématiques et des sciences naturelles pour les écoles primaires ;

- éducation physique et sa didactique, musique et sa didactique, dessin et sa didactique ;

- huit semaines de stage pédagogique et quatre semaines de travail productif.

Le tronc commun des écoles normales supérieures de cycle court comprend philosophie et économie politique, langue étrangère (obligatoire pour le cycle de trois ans, mais pas pour celui de deux ans), éducation physique. Dans la formation de discipline, on enseigne les théories de base, les notions essentielles et les savoir-faire fondamentaux. Il n'y a pas de cours à option en principe, mais des conférences sur des thèmes donnés sont prévues. La formation pédagogique est composée des cours de pédagogie, de psychologie, de didactique, ainsi que huit à douze semaines de stage et d'enquête sociale, y compris deux semaines de stage en responsabilité.

Dans les écoles normales supérieures de cycle long (quatre ans), le tronc commun (politique, langue étrangère, informatique et éducation physique) représente le quart du total tant de l'horaire que des unités de valeurs. La formation disciplinaire se fait par spécialité et occupe une place très importante (70\% du total). Le reste du temps est consacré à la formation pédagogique. Pour les deux dernières parties, la pratique n'est pas du tout négligée et représente 1/10 de l'ensemble, soit environ seize semaines pendant quatre ans.

14 Pour faciliter le stage des normaliens et les recherches pédagogiques, chaque école normale a au moins une école et/ou un jardin d'enfants annexes.

\section{Deux mesures importantes}

15 Ces dernières années, deux mesures importantes concernant la formation ont été prises. Après dix ans d'expériences dans une vingtaine de provinces, le ministère de l'Éducation a publié en 1995 le programme pour former des instituteurs ayant une qualification du premier cycle universitaire. Dans cette école normale de type nouveau, on combine, pour élever le niveau et la qualification des enseignants du primaire, les principales caractéristiques des écoles normales et des écoles normales supérieures de cycle court, et les diplômés des collèges y atteindront, au bout de cinq ans, le niveau de fin d'études secondaires pour l'enseignement général et le niveau du premier cycle universitaire pour une discipline donnée.

$\mathrm{Au}$ niveau des écoles normales supérieures de cycle normal, une révision se fait actuellement dans l'organisation des disciplines et des cours. Le ministère a décidé de diminuer le nombre de départements des disciplines excédentaires, (mathématiques, physique et chimie, en particulier) tout en augmentant celui des disciplines ayant un besoin plus grand et plus pressant afin d'avoir un meilleur équilibre entre l'offre et la demande. Ajouter de nouvelles orientations dans les disciplines existantes, pratiquer le système d'une matière principale plus une matière complémentaire ou de double discipline, sont toutes des mesures ayant le même objectif. Un projet de restructuration des formations pour les futurs enseignants est mis sur pied et a obtenu un consensus presque total. Il consiste à les diviser en cinq grandes parties :

- une formation générale complète et de qualité ;

- une formation pédagogique théorique et pratique ;

- une discipline principale avec des cours de base et une série de cours spécialisés ; 
- une discipline complémentaire choisie par les étudiants et composée des cours de base ;

- de la pratique regroupant stages, exercices militaires, thèse, enquête, travail productif, etc.

\section{Hésitation, contradictions et lacunes} en Chine : hésitation chez certains sur la nécessité de cette formation, contradiction entre son caractère "académique » et «normal » aux yeux de beaucoup, différences régionales très grandes, même croissantes dans le développement en général et dans l'éducation en particulier, lacunes tant qualitatives que quantitatives de cette formation elle-même.

18 La résolution de ces problèmes de genres différents doit être globale et complète et, maintenant, les réformes en cours font apparaitre des perspectives claires tant au niveau théorique et stratégique que pratique et opérationnel.

Au niveau théorique, de plus en plus nombreuse seront ceux qui, dans le débat entre «académique et normal ", reconnaîtront la complémentarité et l'unité de ces deux aspects qui favoriseront un développement sain de la formation des enseignants.

Au niveau stratégique, la tendance est nette :

- la qualification des enseignants se verra élevée et élargie avec la prolongation de la scolarité et la mise en application du système comportant une matière principale plus une matière complémentaire ou une double discipline ;

- la formation ne sera plus limitée aux institutions spécialisées qui, pour leur part, dispenseront des formations autres que celles orientées vers l'enseignement ;

- l'objectif sera plus large, visant non seulement la transmission des connaissances mais aussi, sinon surtout, la formation de l'esprit et des capacités dont aura besoin la société de demain ;

- la formation des enseignants de l'enseignement technique et professionnel entrera dans les écoles normales traditionnelles ;

- la réorganisation de la carte et du réseau de la formation dans le cadre de la province permettra une meilleure utilisation des ressources matérielles et humaines et une intégration de la formation initiale et continue.

21 Au niveau pratique et opérationnel, les différences régionales seront reconnues, même respectées, d'une part et volontairement diminuées, d'autre part, dans la coopération interrégionale et avec le soutien du gouvernement central; l'administration et la gestion se feront d'après les lois et d'une manière plus souple, c'est-à-dire que l'État maintiendra et renforcera les principes définis par les lois, tels que la qualification et le traitement des enseignants, tout en laissant plus de liberté et d'autonomie aux échelons inférieurs, jusqu'aux établissements. On verra dans l'enseignement un renforcement de la formation pédagogique, très importante mais négligée depuis longtemps, avec surtout une amélioration des stages et l'introduction de nouvelles matières, des disciplines carrefour et de nouvelles formes d'enseignement. 


\section{NOTES}

1. La formation continue ne sera pas abordée ici.

\section{RÉSUMÉS}

Un rapide point de vue historique permet d'aborder l'actualité de la formation des enseignants en Chine, caractérisée par des hésitations sur le caractère "normal » ou " académique » de la formation et par des disparités régionales tant quantitatives que qualitatives. La formation, malgré tout, connaît des évolutions très positives dans de nombreux domaines.

A rapid historical review makes it possible to approach the present situation of teacher training in China, characterised by hesitations about the "normal" or academic nature of the training and by regional differences, both as regards quality and quantity, but which, all the same, are undergoing very positive changes in many fields.

Un rápido punto de vista histórico permite abordar la actualidad de la formación de los docentes en China, que se caracteriza por vacilaciones acerca del carácter " normal" o "académico" de la formación y por disparidades regionales no sólo en cantidad sino también en calidad, pero que, a pesar de todo, conoce evoluciones muy positivas en numerosos ámbitos.

\section{INDEX}

Index géographique : Chine

Mots-clés : enseignant, formation des enseignants, programme d'enseignement

\section{AUTEURS}

\section{KECHAO XING}

Professeur, Institut d'Éducation internationale et comparée, Université normale de Beijing (Chine)

\section{YAJUN MENG}

Étudiante en troisième cycle, Université normale de Beijing (Chine) 